\title{
LEA 3: Factor models in population genetics and ecological genomics with $\mathrm{R}$
}

\author{
Clément Gain $\quad$ Olivier François
}

\section{Authors' affiliations:}

Université Grenoble-Alpes, Centre National de la Recherche Scientifique, Grenoble INP, TIMC-IMAG CNRS UMR 5525, 38000 Grenoble, France.

Corresponding author: olivier.francois@univ-grenoble-alpes.fr 


\begin{abstract}
A major objective of evolutionary biology is to understand the processes by which organisms have adapted to various environments, and to predict the response of organisms to new or future conditions. The availability of large genomic and environmental data sets provides an opportunity to address those questions, and the $\mathrm{R}$ package LEA has been introduced to facilitate population and ecological genomic analyses in this context. By using latent factor models, the program computes ancestry coefficients from population genetic data, and performs genotype-environment association analyses with correction for unobserved confounding variables. In this study, we present new functionalities of LEA, which include imputation of missing genotypes, fast algorithms for latent factor mixed models using multivariate predictors for genotype-environment association studies, population differentiation tests for admixed or continuous populations, and estimation of genetic offset based on climate models. The new functionalities are implemented in version 3.0 and higher releases of the package. Using simulated and real data sets, our study provides evaluations and examples of applications, outlining important practical considerations when analyzing ecological genomic data in $\mathrm{R}$.
\end{abstract}

Keywords: Predictive ecological genomics, population structure, genotype-environment association tests, unsupervised machine learning, latent factor models. 


\section{Introduction}

Landscape and ecological genomics attempt to characterize geographic processes underlying the responses of organisms to their environments (Schoville et al., 2012; Sork et al., 2013; Manel \& Holderegger, 2013; Savolainen, Lascoux, \& Merilä, 2013). In these approaches, the recent availability of large genomic and environmental data sets have facilitated the identification of biotic and abiotic factors that influence neutral and adaptive genetic diversity patterns, offering opportunities for researchers to understand those patterns with statistical genetic approaches. Landscape databases include environmental variables such as climate and habitat descriptors which are proxies for geographically heterogeneous selection pressures Fenderson, Kovach, \& Llamas, 2020). Accounting for the confounding effects of demographic processes, local adaptation can be detected at a genomic level by identifying loci which allele frequency exhibits significant association with those environmental variables. Thus, ecological genomic studies could anticipate results from translocation experiments or exposure to future conditions by relying on analyses of population structure and genomic signatures of selection. Many methods and computer programs have been developed to this aim (Rellstab, Gugerli, Eckert, Hancock, \& Holderegger, 2015; Hoban et al., 2016; Forester, Jones, Joost, Landguth, \& Lasky, 2016), and the R package LEA - for Landscape and Ecological Association studies - is one of those programs (Frichot \& François, 2015).

Methods in LEA are based on the statistical framework of latent factor models (Frichot, Schoville, Bouchard, \& François, 2013, Frichot, Mathieu, Trouillon, Bouchard, \& François, 2014). Latent factors are unobserved variables that represent data generated by processes linked to population history, population structure 
and technical or statistical artifacts. Latent representations of large data sets are usually computed as a reduced number of combinations of observed variables, a key step in statistics and in machine learning (Mardia, Kent, \& Bibby, 1979; Murphy, 2012). Technically, the latent factor methods of LEA belong to the class of unsupervised machine learning approaches, and enable users to analyze population structure and detect genomic signatures of local adaptation without assumption on the biological processes that have generated the data. Several variants of latent factor models have been successfully applied in population genetic studies. Examples include estimates of ancestry coefficients with the Bayesian programs structure or tess, computations of eigenvectors in principal component analysis (PCA), uniform manifold approximation and projection for dimension reduction, and factor analysis for ancient DNA samples (Pritchard, Stephens, \& Donnelly, 2000; Caye, Jay, Michel, \& François, 2018; Patterson, Price, \& Reich, 2006; Diaz-Papkovich, Anderson-Trocmé, \& Gravel, 2019; François \& Jay, 2020). In most applications of factor models, projections of individuals on factors reflect their levels of admixture from source populations. In genotypeenvironment association methods, latent factor regression models were introduced to separate variation explained by observed environmental variables from variation explained by unobserved variables (Frichot, Schoville, Bouchard, \& François, 2013). In those regression models, latent factors represent unobserved confounders, and they have less direct interpretations than in ancestry estimation methods. Machine learning algorithms that train models to estimate latent factors are generally computationally efficient with minor loss of statistical accuracy compared to Bayesian Monte-Carlo methods. Thus, they allow their users to increase the volume of data analyzed compared to previous approaches (Caye, Jumentier, Lepeule, \& François, 
2019).

In this study, we present functionalities implemented in LEA version 3 to perform imputation of missing genotypes, improved estimation in latent factor mixed models (LFMM), genome scans for selection based on factor models, and prediction of genetic offset under future environments. Here, future environments should be interpreted as new environments for which we have actual or projected data. Several algorithms processing high-throughput sequencing data do not accept missing genotypes, or handle these values with naive approaches, such as imputation with mean values. Missing data are problematic in genome-wide regression analyses, such as association studies, that have decreased power when genotypes are removed (Marchini \& Howie, 2010). Missing data are also problematic for PCA when it is used for describing structure in the data. See Dray \& Josse (2015) for imputation strategies related to PCA. Imputation of missing data has been an intensive field of statistical research for decades (Van Buuren, 2018). In population genetics, several methods have been proposed to address this issue based on reference genomes. For example, the fastphase model imputes missing genotypes by using linkage disequilibrium and hidden Markov models (Scheet \& Stephens, 2006). Reference genomes are however not available for all organisms, and alternative methods relying on unsupervised machine learning have been considered (Stekhoven \& Bühlmann, 2012, Chi, Zhou, Chen, Del Vecchyo, \& Lange, 2013). LEA 3 implements an imputation algorithm based on factors estimated in the snmf function with a nonnegative matrix factorization approach Lee \& Seung, 1999; Frichot, Mathieu, Trouillon, Bouchard, \& François, 2014). With this approach, missing genotypes are replaced by predicted genotypes in a way that agrees with the inference of population structure. 
The $\mathrm{R}$ package LEA also implements algorithms for LFMMs, which are statistical models used in genotype-environment association studies to identify genomic signatures of adaptation to the local environment (Frichot, Schoville, Bouchard, \& François, 2013). LFMMs optimally separate neutral genetic variation - modelled in the latent factors - from adaptive genetic variation modelled in the effect sizes of environmental covariates. Adaptive loci are expected to be associated with non-null effect sizes, that are tested at each locus in the genomic data. Frichot et al. (2013) used a Bayesian approach and a Markov Chain Monte Carlo (MCMC) algorithm to adjust LFMMs to the data, and this approach was implemented in the $1 \mathrm{fmm}$ function of LEA (Frichot \& François, 2015). Based on least-squares optimization methods (Caye, Jumentier, Lepeule, \& François, 2019), much faster algorithms are now implemented in the lfmm2 function of LEA 3.

In addition to genotype-environment association methods, LEA 3 implements genome scans for selection based on population differentiation - also called outlier tests. Those tests screen a large number of genomic variants across a genome to identify loci that have been affected by diversifying selection. To achieve this objective, they consider the upper tail of the empirical distribution for population differentiation statistics like fixation indices and related measures (Lotterhos \& Whitlock, 2015; Duforet-Frebourg, Luu, Laval, Bazin, \& Blum, 2016; François, Martins, Caye, \& Schoville, 2016). In this context, admixed individuals and genetically continuous populations complicate the use of population differentiation tests. Extensions of population differentiation measures have been recently proposed for samples with admixed individuals (Martins, Caye, Luu, Blum, \& François, 2016). LEA 3 implements the statistics introduced by Martins et al. (2016), and computes significance 
values by comparing the values of those statistics with the genomic background.

Understanding the vulnerability of species and populations to environmental threats is important for developing effective strategies to conserve them (Foden et al., 2019). For a target species, genomic data can be used to evaluate evolutionary potential for future adaptations within a fixed time horizon Sork et al. 2010; Jay et al. 2012; Pauls, Nowak, Bálint, \& Pfenninger, 2013; Aitken \& Whitlock, 2013; Razgour et al., 2019). The genomic approaches exploit the correlation of allele frequencies with geographical variation of the environment to predict mismatches between current and future allele distributions under environmental change projections (Fitzpatrick \& Keller, 2015; Rellstab et al., 2016; Capblancq, Fitzpatrick, Bay, Exposito-Alonso, \& Keller, 2020). In most applications, a set of adaptive loci is first identified from the genomic background using a genotype-environment association method. Then this set of adaptive loci is used to evaluate genetic offset based on another statistical approach (Bay et al., 2018; Ingvarsson \& Bernhardsson, 2020; Waldvogel et al., 2020). LEA 3 implements an approach assuming that effects of the environment are potentially weak but highly polygenic. Using effect sizes instead of significance values, the new approach leverages the entire set of genotyped loci to predict genomic variation under projected conditions. Several examples and simulation studies illustrate the functionalities of LEA 3 below.

\section{New program functionalities}

Imputation of missing data. LEA 3 implements an imputation method based on allele frequencies and ancestry coefficients estimated from its snmf function (Frichot, Mathieu, Trouillon, Bouchard, \& François, 2014). Assuming $n$ diploid organisms 
genotyped at $L$ loci, the snmf algorithm decomposes the $n \times L$ matrix of observed allele frequencies, $P$, in a product of two probabilistic matrices

$$
P \approx Q F
$$

where the coefficients of $P$ take their values in $\{0,1 / 2,1\}$ for diploid organisms (Note: ploidy can be modified in snmf). The matrix $Q$ is similar to the $Q$-matrix of STRUCTURE, representing ancestry coefficients for individuals originating from $K$ source populations (Pritchard, Stephens, \& Donnelly, 2000). The matrix $F$ contains allele frequencies at each locus for each source population. While $P$ may contain some missing values, the product matrix, $Q F$, is always a complete probabilistic matrix. Thus imputation of missing genotypes can be achieved by replacing the missing values with random values sampled from the product matrix distribution. In the same spirit, an alternative option is to pick the most probable genotype based on the largest probabilities in a deterministic fashion. Both random and deterministic procedures were implemented in the impute function of LEA 3.

To evaluate the ability of the impute function to correctly reconstruct missing genotypes, we performed a simulation study of a two-population model with admixture. The simulation model assumes that a haploid population was genotyped at $L$ diallelic loci (values 0 and 1 ) according to the $F$-model (Balding \& Nichols, 1995; Pritchard, Stephens, \& Donnelly, 2000). In the F-model, there is an ancestral gene pool containing alleles of unknown frequencies, $p$, having a uniform distribution over the $L$ loci. At a given time point in the past, the ancestral population split in $K$ subpopulations (here, $K=2$ ), and the subpopulations diverged with genetic drift equal to $F$. Conditional on $p$, the allele frequency at a particular locus in any subpopula- 
tion follows a beta distribution of shape parameters $p(1-F) / F$ and $(1-p)(1-F) / F$. The drift parameter, $F$, controls the fixation index, $F_{\mathrm{ST}}$, with larger values of $F$ causing $F_{\mathrm{ST}}$ 's to be larger. In simulations, the drift parameter was varied between $2 \%$ and $30 \%$. In the model, a third population containing admixed genotypes was also sampled. The admixture rate $\pi_{\text {admix }}$ was varied between $5 \%$ and $50 \%$. For $n=300$ individuals, the number of genotypes, $L$, was varied from 1,000 to 10,000. To create missing genotypes, we removed genotypes at random at a rate, $\pi_{\text {missing }}$, between $10 \%$ and $90 \%$. Ten runs of snmf were performed for each genotypic data set. The best run was retained on the basis of the cross-entropy criterion, and was used for imputation of the missing data. Then we checked whether the removed genotypes were correctly predicted and restored by the deterministic option of the program. The accuracy of reconstruction was measured as the percentage of correctly reconstructed genotypes, and with the root mean squared differences between ancestry estimates computed from the original and reconstructed genotypes. A total of 18,000 program runs for 1,800 data sets were performed. The program snmf was run with $K=2$. The regularization parameter of snmf was increased to $\alpha=100$ (default value $\alpha=10$ ) to account for the relatively small number of loci in the simulations (Frichot, Mathieu, Trouillon, Bouchard, \& François, 2014). Note that parameters used in simulations do not provide any guidelines for snmf in empirical studies. For example, it is always better to increase the number of runs. For their studies, users should refer to original method descriptions, choosing the regularization parameter (and $K$ ) based on the entropy criterion and cross-validation (Owen \& Perry, 2009). Turning to real data, we also simulated missing genotypes in chromosome 5 for 162 European accessions of Arabidopsis thaliana (53,859 single nucleotide polymorphisms, SNPs) Atwell et 
al., 2010). This was done by removing actual genotypes with a rate varying between $10 \%$ and $90 \%$.

Fast latent factor mixed models. LEA 3 (re)implements the LFMM ridge estimation algorithm presented by Caye, Jumentier, Lepeule, \& François (2019). The lfmm2 function handles multivariate environmental data, and estimates locus-specific effect sizes and latent factors by using a least-squares method. The new implementation relies on core functions from the base package of $\mathrm{R}$ which warrants reproducibility of analyses on the long term. To compare $1 \mathrm{fmm} 2$ with the MCMC algorithm programmed in $1 \mathrm{fmm}$, simulations were performed from the LFMM regression model. The model generates continuous values, and a deterministic link function was added in order to obtain haploid genotypes (negative values corresponded to allele 0 and positive values corresponded to allele 1). Following this model, one hundred genotypic matrices were created for $n=100$ individuals genotyped at $L=2,000$ loci. True associations were simulated at 100 loci, with effect sizes between -10 and 10 . A Gaussian environmental variable, $X$, was simulated for each individual, and population structure was modeled by $K=3$ Gaussian latent factors, exhibiting various levels of collinearity with the environmental variable. More precisely, collinearity between $X$ and the latent factors was measured by the coefficient of determination, which varied between $10 \%$ and $80 \%$ in the simulations. High levels of collinearity corresponded to strong confounding effects, and were expected to decrease the power of association tests (Frichot, Schoville, de Villemereuil, Gaggiotti, \& François, 2015b). For the $1 \mathrm{fmm}$ and $\mathrm{lfmm} 2$ tests, power and false discovery rate (FDR) were computed after a Benjamini-Hochberg procedure was applied with an expected FDR level of 
5\% (Benjamini \& Hochberg, 1995). Power was computed as the proportion of true associations that are correctly discovered, and FDR was computed as proportion of false discoveries among the discoveries. In LFMM analyses, the number of factors was set equal to $K=3$, corresponding to a recommended value obtained from the elbow in PCA scree-plots for the genetic data.

In a second series of experiments, we compared the relative performances of $1 \mathrm{fmm} 2$ with genome scans based on redundancy analysis (RDA) (Mardia, Kent, \& Bibby, 1979; Forester, Lasky, Wagner, \& Urban, 2018). To this objective, we re-analyzed one hundred simulated data sets from Capblancq, Luu, Blum, \& Bazin (2018). Each data set was obtained from forward simulations of biologically realistic scenarios with weak population structure, and contained $n=640$ diploid individuals genotyped at $L=1,000$ SNPs. In these scenarios, three quantitative traits were controlled by 10 unlinked loci (QTLs). Ten environmental variables were also created. The first three environmental variables determined selective pressure on the traits, whereas the other ones had no effect on the phenotypes. Power and FDR were computed for lfmm2, RDA and partial RDA. Partial RDA was introduced to correct for confounding due to population structure and to provide a fair comparison of algorithms by conditioning RDA on latent factors estimated by lfmm2. To compare the results with (Capblancq, Luu, Blum, \& Bazin, 2018), we used the parameters and the computer codes of their study, performing association analyses with principal components built on environmental variables and setting the expected FDR level to $10 \%$.

Identifying outlier loci with latent factor models. Statistical tests to identify loci associated with extreme values of population differentiation statistics were 
implemented in the snmf . pvalues function of LEA 3 (Martins, Caye, Luu, Blum, \& François, 2016). For $K$ ancestral populations, the population differentiation statistics were defined at each locus as follows

$$
F_{\mathrm{ST}}=1-\frac{\sum_{k=1}^{K} q_{k} f_{k}\left(1-f_{k}\right)}{f(1-f)},
$$

where $q_{k}, f_{k}$ and $f$ were obtained from the $Q$ and $F$ estimates computed by the snmf function. The quantity $q_{k}$ was equal to the average ancestry coefficient across individuals, $f_{k}$ was equal to the allele frequency in population $k$, and we set $f=\sum_{k=1}^{K} q_{k} f_{k}$. Based on those modified $F_{\mathrm{ST}}$ statistics, significance values were computed by using the Fisher distribution. Fisher tests are similar to chi-squared tests, which have been extensively studied both for snmf and for PCA (Martins, Caye, Luu, Blum, \& François, 2016; Duforet-Frebourg, Luu, Laval, Bazin, \& Blum, 2016, Chen, Lee, Zhu, Benyamin, \& Robinson, 2016; Galinsky et al., 2016). With PCA, genome scans were performed by fitting a regression model for each SNP, for which the response variable is the SNP frequency and the explanatory variables are the $K-1$ first PCs of the genetic data. We illustrated the results of outlier tests implemented in the snmf .pvalues function by re-analyzing SNP data for 49 accessions from Scandinavian lines of Arabidopsis thaliana (Atwell et al. 2010). Filtering loci for minor allele frequency greater than 5\% resulted in 205,417 SNPs across the five chromosomes. We compared the results of the tests with tests based on PCA loadings and with LFMMs considering latitude as an explanatory variable which correlates with population structure $(K=2)$. 
Genetic offset statistics. LEA 3 allows computing predictive measures of genetic offset based on future environmental data. This section presents a brief outline of the theory underlying the computation of genetic offsets, sufficient for the interpretation of the program outputs. For a single population, the offset statistic measures the divergence between allele frequencies in current conditions and in a fictive population harboring frequencies corresponding to future (or new) conditions. For this population, the function considers two sets of environmental variables, $\mathbf{X}_{\text {current }}$ and

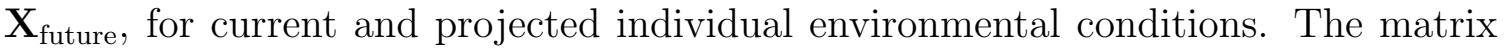
of current variables is first used to fit an LFMM, and the fitted model is then applied to the new data for the prediction of responses. Technically, two genetic matrices, $\mathbf{Y}_{\text {fit }}$ and $\mathbf{Y}_{\text {pred }}$ are constructed

$$
\mathbf{Y}_{\text {fit }}=\mathbf{X}_{\text {current }} \mathbf{B}^{T}+\mathbf{U} \mathbf{V}^{T}
$$

and

$$
\mathbf{Y}_{\text {pred }}=\mathbf{X}_{\text {future }} \mathbf{B}^{T}+\mathbf{U V}^{T}
$$

where $\mathbf{B}, \mathbf{U}$, and $\mathbf{V}$ are the effect size, factor and loading matrices adjusted by the lfmm2 algorithm from the current data. In matrix notation, $\mathbf{B}^{T}$ denotes the transpose of the matrix $\mathbf{B}$. Then we considered $\sigma_{\text {pred }}$ and $\sigma_{\text {fit }}$ the largest singular values of the matrices $\mathbf{Y}_{\text {pred }}$ and $\mathbf{Y}_{\text {fit }}$, and $\sigma_{\text {pred+fit }}$ the largest singular value of the concatenated matrix $\left(\mathbf{Y}_{\text {pred }}, \mathbf{Y}_{\text {fit }}\right)^{T}$. The singular values were computed with the svd function of R, after all matrices were standardized. A genetic offset, $F_{\text {offset }}$, is computed as follows 


$$
1-F_{\text {offset }}=\frac{1-\sigma_{\text {pred+fit }}^{2}}{1-\left(\sigma_{\text {pred }}^{2}+\sigma_{\text {fit }}^{2}\right) / 2} .
$$

This definition of genetic offset is justified by the spectral analysis of population structure (Patterson, Price, \& Reich, 2006). According to the genealogical interpretation of singular values (McVean, 2009; Bryc, Bryc, \& Silverstein, 2013), the quantity $F_{\text {offset }}$ is similar to a drift coefficient in a two-population model (Slatkin, 1991). The genetic offset measures the amount of genetic drift separating the population adapted to the current range of environmental variables to the fictive population adapted to the range of projected variables. In comparison to existing statistics (Fitzpatrick \& Keller, 2015; Rellstab et al., 2016), the new measures do not select a particular subset of outlier loci but instead integrate over effects of environment at the genome scale, accounting for population structure. The statistics were implemented in the genetic. offset function of LEA 3.

To illustrate the function, we considered a subset of the 1,001 Genomes data for the plant species Arabidopsis thaliana (Alonso-Blanco et al., 2016). Two-hundred forty-one accessions from Southern, Central and Northern Sweden were extracted from the database. A matrix of SNP genotypes was obtained by considering variants with minor allele frequency greater than $5 \%$ and a density of variants around one SNP every 500 bp (334,946 SNPs). Because the genetic offset is a population statistic, we needed to cluster the individuals into groups. The individuals were clustered in eight groups after a preliminary analysis of population structure with snmf and on the basis of geographic proximity. To account for residual population structure in each of the eight groups, we chose $K=4$ factors in the LFMM predictions (a conservative choice having minor impact on the results). Global climate and 
(a)

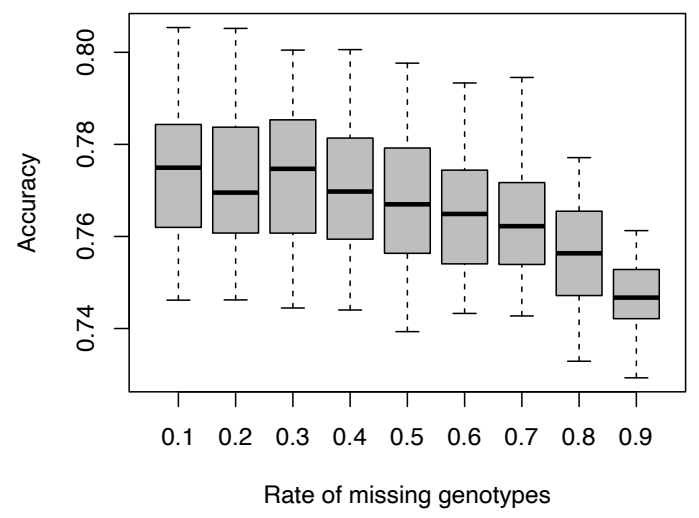

(b)

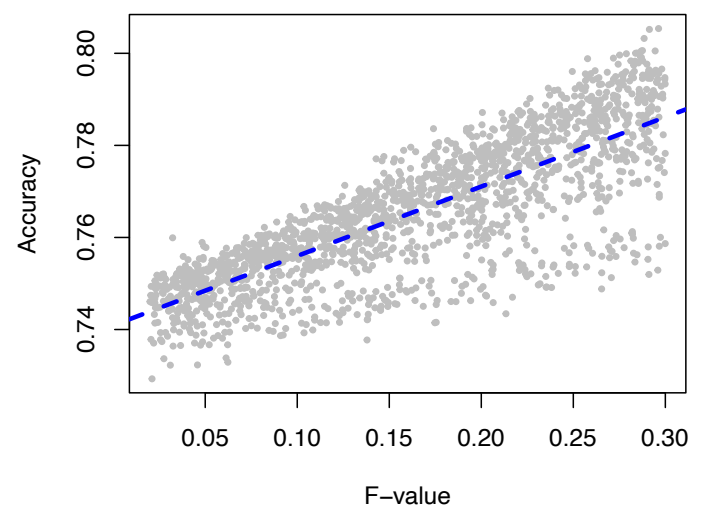

Figure 1. Imputation of missing data. Simulations from a two-population Fmodel with admixture. a. Accuracy of reconstructed genotypes as a function of the rate of missing genotypes. b. Accuracy of reconstructed genotypes as a function of the drift parameter $F$.

weather data corresponding to individual geographic coordinates were downloaded from the WorldClim database (https://worldclim.org). Eighteen bioclimatic variables, derived from the monthly temperature and rainfall values, were considered as representing the current environmental matrix. Projected environmental variables were obtained from three Representative Concentration Pathway (RCP) trajectories adopted by the IPCC fifth Assessment Report (2014). The pathways described different climate futures, corresponding to RCP 2.6, RCP 4.5, and RCP 8.5 (70 years) (IPCC, 2014).

\section{Results}

Imputation of missing data. To evaluate the ability of the impute function to correctly reconstruct missing genotypes, we analyzed simulated genotypes from two 
populations with divergence and admixture. Over all data sets, the reconstruction accuracy for a missing genotype was around 76.5\%, with a standard deviation of $14.1 \%$ (Figure 1). It was similar to the accuracy of reconstruction of non-missing genotypes by matrix factorization (mean around $77.5 \%$ with a standard deviation of $12.3 \%$ ). The root mean squared differences between ancestry coefficients estimated from true and imputed genotypes were of the same order as the root mean squared differences between ancestry coefficients computed from distinct runs on the full data set $($ mean $=5.2 \%$ with a standard deviation of $3.1 \%$ ). These results show that the reconstructed matrices were statistically similar to the original ones having no missing data. Significant association of accuracy with the proportion of missing data was observed (Multiple $R$-squared: $0.27, F$-statistic: 660.3 on 1 and $1798 \mathrm{df}, P$ lower than 2.2e-16). The results indicated that imputation was robust to removing a large fraction of genotypes, up to $30 \%$ (Figure 1A). Significant association was also observed between accuracy and the drift parameter (Multiple $R$-squared: 0.65, $F$-statistic: 3291 on 1 and 1798 df, $P$ lower than 2.2e-16), indicating that imputation performed better when the population split occurred at older dates (Figure 1B). There were weak effects of the number of loci considered and admixture rate, leading to non-significant $p$-values. We eventually tested the effect of interaction between the simulation parameters, and found that accuracy was best explained by the following combination of simulation parameters (Multiple R-squared: 0.92, F-statistic: 5125 on 4 and $1795 \mathrm{df}): 0.747+0.221 F-0.010 \pi_{\text {missing }}-0.106 F \times \pi_{\text {missing }}-0.073 F \times \pi_{\text {admix }}$. Accuracy increased with larger values of $F$ but not so much when the rate of missing values or the admixture rate increased at the same time. Next, we considered missing genotypes in European accessions of Arabidopsis thaliana by removing a proportion 
$\pi_{\text {missing }}$ of actual genotypes. Using a model with $K=6$ clusters, the accuracy of reconstructed genotypes varied approximately as $0.784-0.009 \pi_{\text {missing }}-0.050 \pi_{\text {missing }}^{3}$ (Multiple $R$-squared: 0.99). For $10 \%$ missing values, the total number of correct genotypes in the imputed data was around $98 \%$ of the Arabidopsis data, which had no missing genotypes. For $80 \%$ missing values, the total number of correct genotypes in the imputed data was around $80 \%$ of the original data.

Latent factor mixed models. To compare the statistical performances of the lfmm and $1 \mathrm{fmm} 2$ estimation algorithms, 100 genotypic matrices were simulated, based on the common generative model for both approaches (Figure 2). The average FDR was around its expected value of $5 \%$ for 1 fmm (mean value $6.4 \%$, Figure $2 \mathrm{~A}$ ). The average FDR was significantly lower for 1 fmm2 (mean value $3 \%, t$-test $=6.88, P=$ 1.2e-10), showing that the least-squares algorithm was more accurate than the MCMC algorithm when simulations were performed under the generative model. For those simulations, the two versions had equivalent power to reject loci with null effect sizes (Figure 2B). Power was generally high (greater than 90\%) when collinearity between population structure and the environmental variable was moderate, corresponding to values of the coefficient of determination lower than $60 \%$. A sharp decrease in power was observed when the coefficient of determination reached values higher than $60 \%$.

Next, we compared the relative performances of $1 \mathrm{fmm} 2$ with approaches based on RDA and partial RDA, resuming simulations conducted in Capblancq, Luu, Blum, \& Bazin, 2018) (Figure 3). Power and FDR obtained with RDA and partial RDA were highly similar, in agreement with weak population structure observed in the simulations (Capblancq, Luu, Blum, \& Bazin, 2018). Power was close to $100 \%$ for all 
(a)

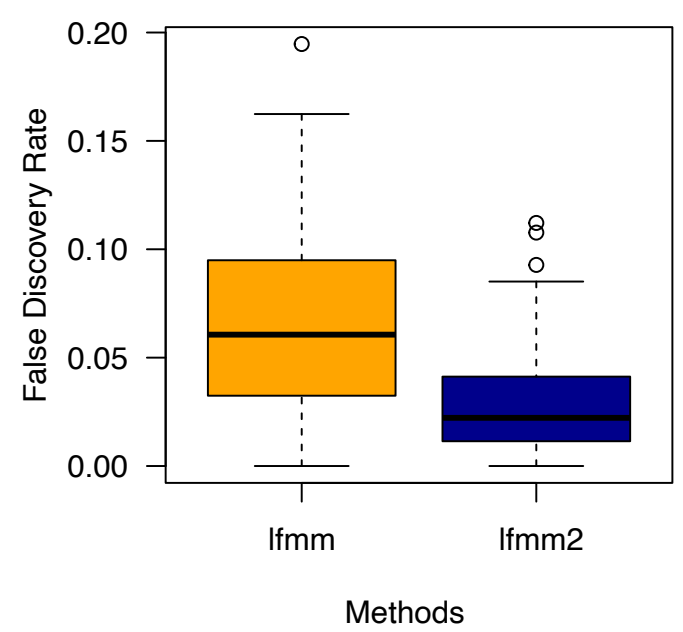

(b)

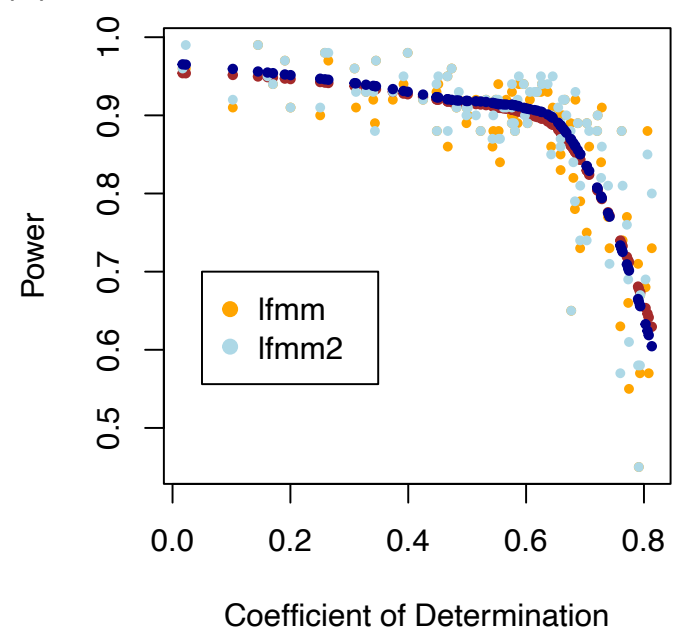

Figure 2. Comparisons of $\mathrm{lfmm}$ (MCMC) with $\mathrm{lfmm} 2$ (least squares). a. False discovery rates observed for an expected FDR of $5 \%$. b. Power to reject null effect sizes. The coefficient of determination is the proportion of environmental variation explained by the hidden factors in the simulated data. The dark blue and (hidden) brown points correspond to local regressions of the power values. 
(a)

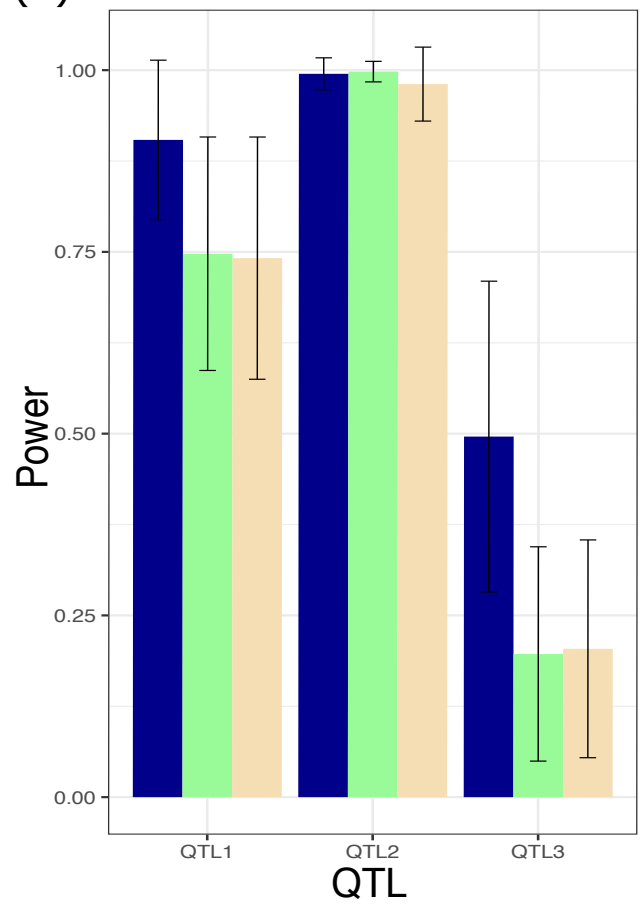

(b)

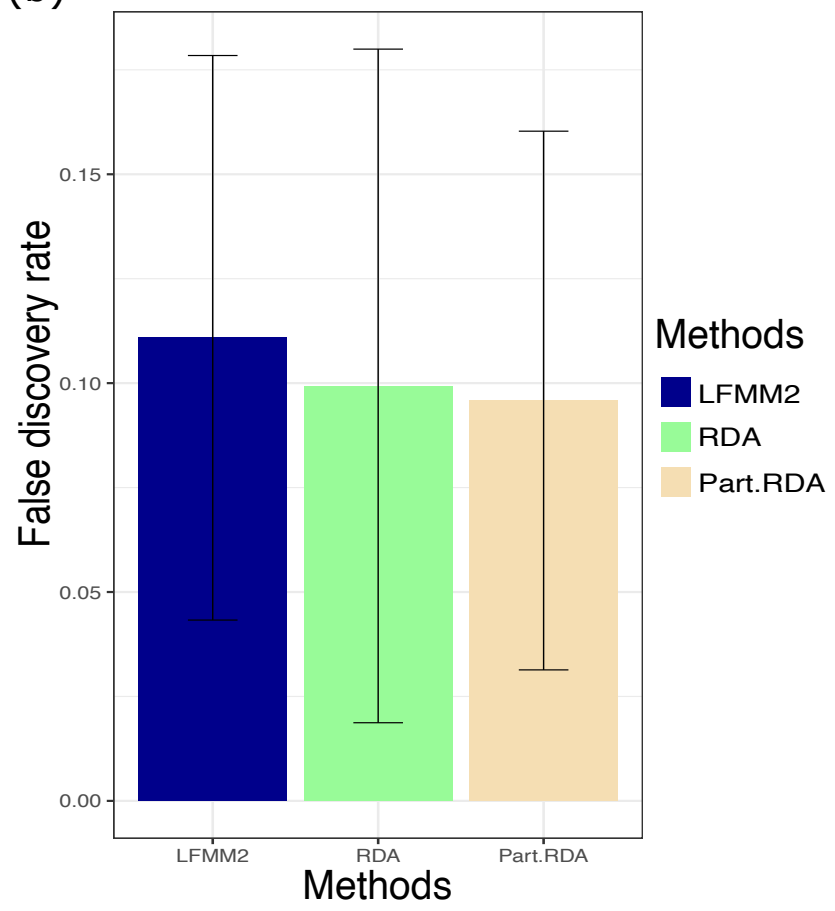

Figure 3. Comparisons of $1 \mathrm{fmm} 2$ with RDA and partial RDA. Simulations resumed from (Capblancq et al. 2018). a. Power to detect loci under selection, b. False discovery rate for a controlled FDR level of 10\%. Partial RDA (Part. RDA) includes corrections based on LFMM latent factors.

10 SNPs in QTL2 with all methods. FDRs were around their expected values for all methods (10\%). Overall, lfmm2 had increased power compared to RDA approaches for SNPs in QTL1 (mean value for $1 \mathrm{fmm} 2=90 \%$, mean value for $\mathrm{RDA}=75 \%, t$-test $=8.01, P=1.602 \mathrm{e}-13$ ) and QTL3 (mean value for $1 \mathrm{fmm} 2: 50 \%$, mean value for RDA: $20 \%, t$-test $=11.45, P$ lower than $2.2 \mathrm{e}-16)$, contrasting with results for $1 \mathrm{fmm}$ reported in the previous study.

Identifying outlier loci with latent factor models. We illustrated outlier tests based on population differentiation statistics introduced in Martins, Caye, Luu, Blum, 

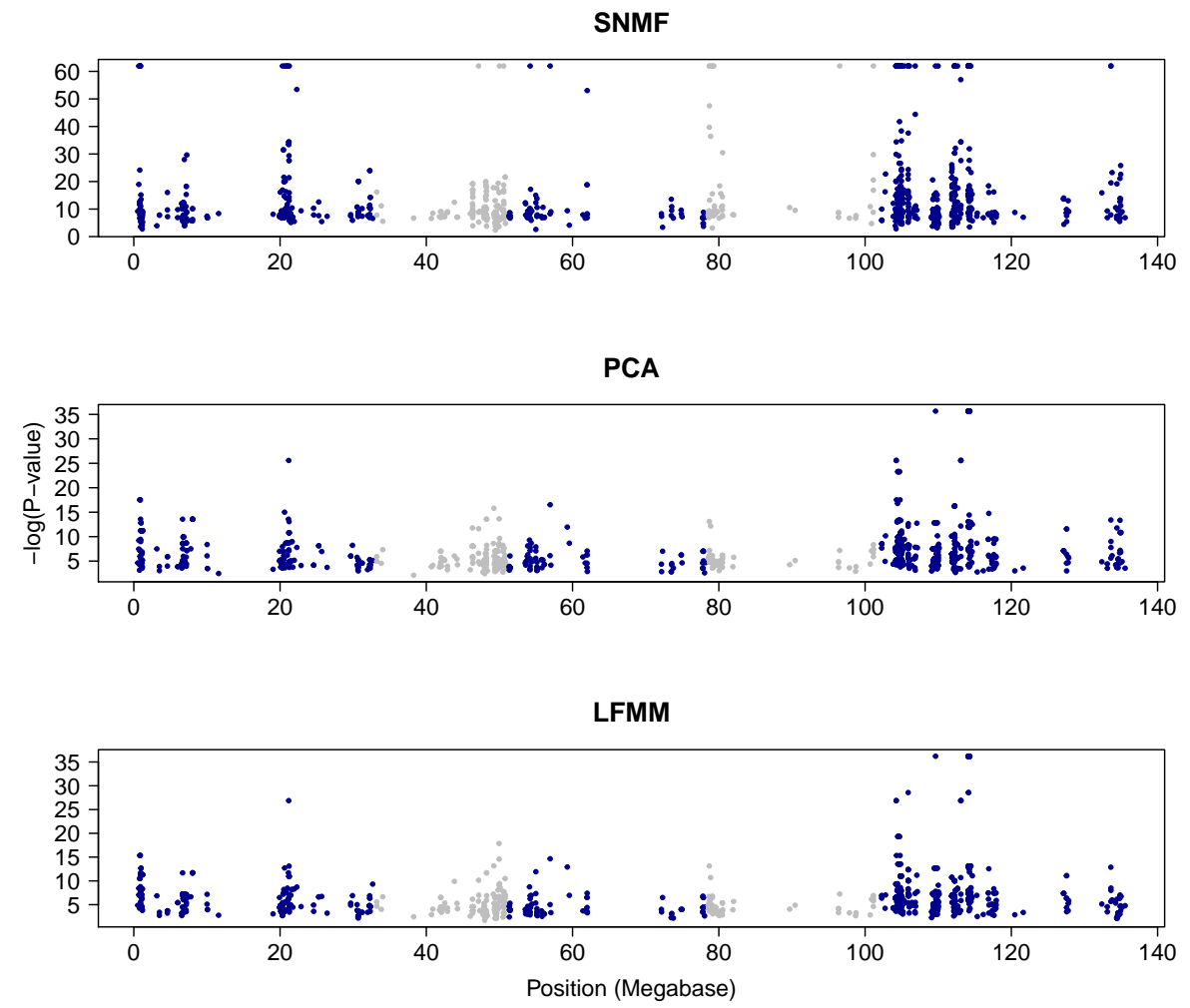

Figure 4. Genome scans for selection. SNP genotypes of forty-nine accessions of Arabidopsis thaliana from Northern and Southern Sweden analyzed with snmf, PCA, and $1 \mathrm{fmm} 2$ with latitude used as an explanatory variable. Only loci with -log $P$ greater than the Bonferroni threshold in one method $(=6.61)$ are shown. See Figure $\mathrm{S} 4$ for a focus on the region around $113 \mathrm{Mb}$. 
\& François (2016) by re-analyzing SNP data for 49 Scandinavian accessions Arabidopsis thaliana with snmf.pvalues, with PCA loadings and with lfmm2. The samples were divided in two geographic groups located in southern (37 individuals) and in northern Sweden (12 individuals). Latitude was used as an environmental variable in lfmm2. Although the histograms of $P$-values exhibited similar shapes for all methods (Figure S1), differences were observed in the tails of the test statistics. The $P$-values were lower in snmf than in $1 \mathrm{fmm} 2$ and PCA (Figure 4). The Pearson correlation between snmf and PCA significance values was high, around 95\%, and greater than the correlation between PCA and lfmm2 values (91\%). These results can be explained by a high correlation between ancestry coefficients and PC1 scores (Figure S2). Despite the differences in the tails of distributions, genome scans based on snmf, PCA and lfmm2 hit the same genomic regions in chromosomes 1 and 5 of the plant, and the ranking of association levels were comparable in the three approaches. After Bonferroni correction, there were 1,013 significant hits at the 5\% nominal level for snmf, 485 for PCA, and 459 for $1 \mathrm{fmm} 2$, corresponding to around 50 genomic regions (Figure 4). The top hits for snmf, with significance values above 50, did not reach the highest levels in the PCA and LFMM tests. Those 129 hits corresponded to SNPs with allelic frequencies close to fixation either in the southern or the northern group (Figure S3). Focusing on a region of chromosome 5 around $113 \mathrm{Mb}$ which was enriched in top hits, we estimated heterozygozity along the chromosome (Figure S4). This analysis provided evidence that a few loci exhibit signatures of selective sweep, more often in the northern group than in the southern group. 
Genetic offset statistics. Leveraging multivariate environmental data analysis with lfmm2, we illustrated the computation of genetic offset statistics for Scandinavian populations of $A$. thaliana (241 individuals) obtained from the prediction of eighteen bioclimatic variables based on three RCP trajectories (70 years). In LEA 3 , genetic offset has the same interpretation as $F_{\mathrm{ST}}$, measuring the percentage of inbreeding in the population formed by the union of the current and projected samples. Under RCP 2.6, the genetic offset statistics ranged between $0 \%$ and $56 \%$, with a mean value around 29\% (Figure 5). For RCP 4.5 and 8.5, the maximum ranges stretched to $74 \%$ and $79 \%$ with mean values around $51 \%$ and $60 \%$ respectively (Figure 5). In RCP 2.6, the most exposed populations were at latitude around $50^{\circ} \mathrm{N}$ in Southern Scandinavia. Under RCP 4.5 and RCP 8.5, the most exposed populations were in the north at latitude around $62^{\circ}-64^{\circ} \mathrm{N}$. The risks were poorly explained by latitude alone $\left(R^{2}\right.$ less than $\left.10 \%\right)$, but they were consistent with the principal components of projected climatic variables under the considered scenarios (PCs shown in Figures S5-S6).

\section{Discussion}

Modern molecular ecology studies make extensive use of unsupervised statistical methods for their analysis of large population genomic data (Hendricks et al., 2018; Paradis et al., 2017). The $\mathrm{R}$ program LEA provides an integrated suite of functions for running such analyses, including model-free inference of population structure and genome scans for signatures of local adaptation. This computer note presented functionalities implemented in version 3.1 of the computer program, and surveyed some applications of factorial methods in molecular ecology and ecological 

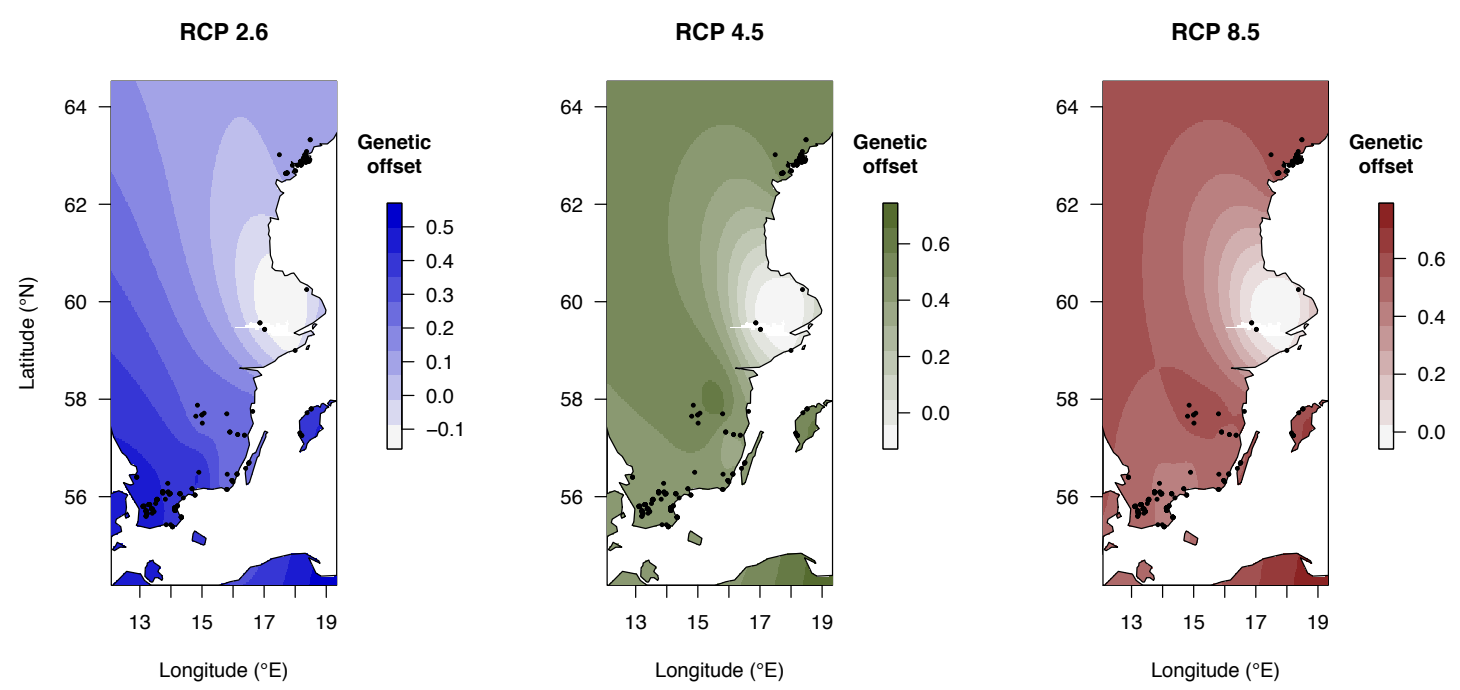

Figure 5. Genetic offset for Scandinavian populations of Arabidopsis thaliana. Genetic offset computed from projections of 18 bioclimatic variables according to three climate models RCP 2.6, 4.5 and RCP 8.5 (70 years). The genetic offset statistics were computed for 8 population samples. The values were interpolated by using the kriging algorithm implemented in fields 10.2 at the 241 sampling sites represented as black dots (Nychka et al. 2017). 
genomics. Since the first release of the package, new factor methods have been developed and integrated in the development version of the package. These new methods encompass genome scans based on population differentiation statistics and genotype-environment association studies, which have been evaluated in previous studies (Martins, Caye, Luu, Blum, \& François, 2016; Caye, Jumentier, Lepeule, \& François, 2019). The inclusion of those methods in LEA will facilitate their applications by $\mathrm{R}$ users, avoiding unnecessary coding or data formatting. Additional functionalities, including imputation of missing data and predictive values of genetic offset increase the range of applications of the package. A drawback of latent representations built in machine learning algorithms is to favor prediction to the detriment of interpretation (Lipton, 2018). With latent factor models, interpretation of results in terms of standard population genetic concepts, such as ancestry coefficients, population differentiation and genetic drift is preserved, as well as their appropriateness for predictive objectives.

Concerning missing data, the method implemented in LEA 3 is a novel approach, based on matrix factorization and on ancestry coefficients estimated by snmf (Frichot, Mathieu, Trouillon, Bouchard, \& François, 2014). The matrix factorization technique has been frequently employed in machine learning applications where it has been successful in collective filtering and recommender systems Koren, Bell, \& Volinsky, 2009). The approach is conceptually similar to the imputation methods used by Bayesian programs like structure (Pritchard, Stephens, \& Donnelly, 2000). Imputation based on snmf factors is consistent with respect to the allele frequencies in the 'ancestral' populations. Thus the method is appropriate for genotype-environment association studies and other applications using ancestral allele frequency informa- 
tion. An advantage of matrix factorization approaches is to be efficient without reference genomes. For example, imputation based on matrix factorization decreased the proportion of missing data from $10 \%$ to $2 \%$ in Arabidopsis thaliana simulations. While the limits on imputation are generally poorly understood and specific to each data set, we encourage users to remove a small proportion of non-missing genotypes (for example, 1\%) and check the validity of their imputation results in preliminary analytical stages of their empirical studies.

Regarding genotype-environment association studies, least squares estimates for latent factor regression models were implemented in the $1 \mathrm{fmm} 2$ function (Caye, Jumentier, Lepeule, \& François, 2019). The function 1 fmm2 extends 1 fmm by allowing multivariate environmental data, which is particularly useful for predictive applications. The main improvements over $\mathrm{lfmm}$ are substantial savings of computer resources thanks to an exact algorithm rather than a Monte Carlo method. Although the $\mathrm{lfmm} 2$ and $\mathrm{lfmm}$ methods assumes slightly different sparsity conditions on effect size estimates, we found that they were equivalent in terms of sensitivity, reaching high levels of true positive rates when the coefficient measuring collinearity between environmental variables and latent factors was below 60\%. An abrupt decrease in power was however observed for coefficients greater than $60 \%$. These results suggests that users should attempt to evaluate the determination coefficient a priori from the first principal components of the genomic data or a posteriori from factors estimated by the methods. After reanalyzing simulated data sets from (Capblancq, Luu, Blum, $\&$ Bazin, 2018), we found that the multivariate version of $1 \mathrm{fmm} 2$ compared favorably with genome scans based on redundancy analysis. The explanation for improvement over the results reported by Capblancq, Luu, Blum, \& Bazin (2018) is that 
our analysis included more environmental information than did the previous study, exemplifying the benefit of using multivariate environmental data in LFMMs.

Population differentiation tests are often used in assessing genomic signatures of local adaptation. For Arabidopsis thaliana, population differentiation tests based on snmf, PCA and association with latitude highlighted similar genomic regions. Because those regression methods exhibit almost maximal collinearity between population structure and the explanatory variable, the interpretation of genome scans may be difficult, and it is complicated by the demographic history of Scandinavian A. thaliana populations (Huber, Nordborg, Hermisson, \& Hellmann, 2014; Lee et al. 2017). A large fraction of the SNPs discovered by the genome scan methods might have resulted from neutral demographic processes or from linked selection. Choosing environmental variables not co-varying with population structure could alleviate the problem in genotype-environment association methods (François, Martins, Caye, \& Schoville, 2016). With dense genomic data, we also suggest that population differentiation tests should be coupled with methods to detect selective sweeps (Vatsiou, Bazin, \& Gaggiotti, 2016). For example, when we focused on a region enriched in snmf top hits, the analysis of heterozygozity along the fifth chromosome supported that some hits exhibited convincing signatures of selection, in particular in the northern group.

Following recent approaches in predictive ecological genomics (Bay et al., 2018; Fitzpatrick \& Keller, 2015), LEA 3 can compute genetic offset statistics based on models of association between environmental variables and allele frequencies. The new statistic accounts for potentially small effects of the environment that are spread across the genome and the offset computation assumes that changes in population 
structure will occur only through modification of adaptive allele. In addition, genetic offset has an interpretation similar to a population differentiation statistic, measuring drift between allele frequencies in populations under current and future conditions. According to (Skoglund, Sjödin, Skoglund, Lascoux, \& Jakobsson, 2014, Frachon et al., 2017), the values of this drift coefficient can be converted in generation time using the equation, $t=4 N_{\mathrm{e}} F_{\text {offset }} /\left(1-F_{\text {offset }}\right)$, where $N_{\mathrm{e}}$ is the effective population size. For Scandinavian populations of $A$. thaliana, relatively large values of $N_{\mathrm{e}}$ have been reported, around $N_{\mathrm{e}} \approx 1,000$ (Lundemo, Falahati-Anbaran, \& Stenøien, 2009). For these values, an offset statistic around 30-70\% indicates that the evolutionary time required for adaptation to predicted climates will be around four thousands generations. While estimates may be biased upward by having removed rare variants from the analysis, a robust result is that genetic offset was higher in northern populations under the most extreme RCP scenario.

Computer programs evolve and upgrading LEA to include recent developments of latent factors in ecological genomics was necessary. The functions implemented in LEA 3 are built on classes of objects that are fully consistent with the previous versions of the program, so that users will adapt easily to the new version. Examples included in the program documentation and in a tutorial are available from www. bioconductor.org for new users to learn the program quickly.

Acknowledgments. The authors are grateful to the organizers of the special issue for their invitation to submit a manuscript. They thank the reviewers for useful and constructive comments that improved the presentation of the manuscript. This work 
received support from the "Predictive Ecological Genomics" project (MIAI@GrenobleAlpes, ANR-19-P3IA-0003).

\section{References}

Aitken, S. N., \& Whitlock, M. C. (2013). Assisted gene flow to facilitate local adaptation to climate change. Annual Review of Ecology, Evolution, and Systematics, 44, 367-388.

Alonso-Blanco, C., Andrade, J., Becker, C., Bemm, F., Bergelson, J., Borgwardt, K. M., ... The 1001 Genomes Consortium (2016). 1,135 genomes reveal the global pattern of polymorphism in Arabidopsis thaliana. Cell, 166, 481-491.

Atwell, S., Huang, Y. S., Vilhjálmsson, B. J., Willems, G., Horton, M., Li, Y., ... Nordborg, M. (2010). Genome-wide association study of 107 phenotypes in Arabidopsis thaliana inbred lines. Nature, 465, 627-631.

Balding, D. J. \& Nichols, R. A. (1995). A method for quantifying differentiation between populations at multi-allelic loci and its implications for investigating identity and paternity. Genetica, 96, 3-12.

Bay, R. A., Harrigan, R. J., Le Underwood, V., Gibbs, H. L., Smith, T. B., \& Ruegg, K. (2018). Genomic signals of selection predict climate-driven population declines in a migratory bird. Science, 359, 83-86.

Benjamini, Y.,\& Hochberg, Y. (1995). Controlling the false discovery rate: a practical and powerful approach to multiple testing. Journal of the Royal Statistical Society: Series B, 57, 289-300. 
Bryc, K., Bryc, W., \& Silverstein, J. W. (2013). Separation of the largest eigenvalues in eigenanalysis of genotype data from discrete subpopulations. Theoretical Population Biology, 89, 34-43.

Caye, K., Jay, F., Michel, O., \& François, O. (2018). Fast inference of individual admixture coefficients using geographic data. The Annals of Applied Statistics, 12, $586-608$.

Caye, K., Jumentier, B., Lepeule, J., \& François, O. (2019). LFMM 2: Fast and accurate inference of gene-environment associations in genome-wide studies. Molecular Biology and Evolution, 36, 852-860.

Capblancq, T., Luu, K., Blum, M. G., \& Bazin, E. (2018). Evaluation of redundancy analysis to identify signatures of local adaptation. Molecular Ecology Resources, 18, 1223-1233.

Capblancq, T., Fitzpatrick, M. C., Bay, R. A., Exposito-Alonso, M., \& Keller, S. R. (2020). Genomic prediction of (mal) adaptation across current and future climatic landscapes. Annual Review of Ecology, Evolution, and Systematics, 51, doi.org/10.1146.

Chen, G. B., Lee, S. H., Zhu, Z. X., Benyamin, B., \& Robinson, M. R. (2016). EigenGWAS: finding loci under selection through genome-wide association studies of eigenvectors in structured populations. Heredity, 117, 51-61.

Chi, E. C., Zhou, H., Chen, G. K., Del Vecchyo, D. O., \& Lange, K. (2013). Genotype imputation via matrix completion. Genome Research, 23, 509-518. 
Diaz-Papkovich, A., Anderson-Trocmé, L., \& Gravel, S. (2019). UMAP reveals cryptic population structure and phenotype heterogeneity in large genomic cohorts. PLoS Genetics, 15, e1008432.

Dray, S., \& Josse, J. (2015). Principal component analysis with missing values: a comparative survey of methods. Plant Ecology, 216, 657-667.

Duforet-Frebourg, N., Luu, K., Laval, G., Bazin, E., \& Blum, M. G. (2016). Detecting genomic signatures of natural selection with principal component analysis: application to the 1000 genomes data. Molecular Biology and Evolution, 33, 10821093.

Fenderson, L. E., Kovach, A. I., \& Llamas, B. (2020). Spatiotemporal landscape genetics: Investigating ecology and evolution through space and time. Molecular Ecology, 29, 218-246.

Fitzpatrick, M. C., \& Keller, S. R. (2015). Ecological genomics meets communitylevel modelling of biodiversity: Mapping the genomic landscape of current and future environmental adaptation. Ecology Letters, 18, 1-16.

Foden, W. B., Young, B. E., Akçakaya, H. R., Garcia, R. A., Hoffmann, A. A., Stein, B. A., ... Huntley, B. (2019). Climate change vulnerability assessment of species. Wiley interdisciplinary reviews: climate change, 10, e551.

Forester, B. R., Jones, M. R., Joost, S., Landguth, E. L., \& Lasky, J. R. (2016). Detecting spatial genetic signatures of local adaptation in heterogeneous landscapes. Molecular Ecology, 25, 104-120. 
Forester, B. R., Lasky, J. R., Wagner, H. H., \& Urban, D. L. (2018). Comparing methods for detecting multilocus adaptation with multivariate genotype-environment associations. Molecular Ecology, 27, 2215-2233.

Frachon, L., Libourel, C., Villoutreix, R., Carrère, S., Glorieux, C., Huard-Chauveau, C., ... Roux, F. (2017). Intermediate degrees of synergistic pleiotropy drive adaptive evolution in ecological time. Nature Ecology and Evolution, 1, 1551-1561.

François, O., Martins, H., Caye, K., \& Schoville, S. D. (2016). Controlling false discoveries in genome scans for selection. Molecular Ecology, 25, 454-469.

François, O., \& Jay, F. (2020). Factor analysis of ancient population genomic samples. Nature Communications, 11, in press.

Frichot, E., Schoville, S. D., Bouchard, G., \& François, O. (2013). Testing for associations between loci and environmental gradients using latent factor mixed models. Molecular Biology and Evolution, 30, 1687-1699.

Frichot, E., Mathieu, F., Trouillon, T., Bouchard, G., \& François, O. (2014). Fast and efficient estimation of individual ancestry coefficients. Genetics, 196, 973-983.

Frichot, E., \& François, O. (2015). LEA: An R package for landscape and ecological association studies. Methods in Ecology and Evolution, 6, 925-929.

Frichot, E., Schoville, S. D., de Villemereuil, P., Gaggiotti, O. E., \& François, O. (2015). Detecting adaptive evolution based on association with ecological gradients: Orientation matters! Heredity, 115, 22-28. 
Galinsky, K. J., Bhatia, G., Loh, P. R., Georgiev, S., Mukherjee, S., Patterson, N. J., \& Price, A. L. (2016). Fast principal-component analysis reveals convergent evolution of $A D H 1 B$ in Europe and East Asia. The American Journal of Human Genetics, 98, 456-472.

Hendricks, S., Anderson, E. C., Antao, T., Bernatchez, L., Forester, B. R., Garner, B., ... Luikart, G. (2018). Recent advances in conservation and population genomics data analysis. Evolutionary Applications, 11, 1197-1211.

Hoban, S., Kelley, J. L., Lotterhos, K. E., Antolin, M. F., Bradburd, G., Lowry, D. B., ... Whitlock, M. C. (2016). Finding the genomic basis of local adaptation: pitfalls, practical solutions, and future directions. The American Naturalist, 188, 379-397.

Huber, C. D., Nordborg, M., Hermisson, J., \& Hellmann, I. (2014). Keeping it local: evidence for positive selection in Swedish Arabidopsis thaliana. Molecular Biology and Evolution, 31, 3026-3039.

Ingvarsson, P. K., \& Bernhardsson, C. (2020). Genome-wide signatures of environmental adaptation in European aspen (Populus tremula) under current and future climate conditions. Evolutionary Applications, 13, 132-142.

Intergovernmental Panel on Climate Change (2014). Climate Change 2014: Synthesis Report. Contribution of Working Groups I, II and III to the Fifth Assessment Report of the Intergovernmental Panel on Climate Change, IPCC, Geneva, Switzerland. 
Jay, F., Manel, S., Alvarez, N., Durand, E. Y., Thuiller, W., Holderegger, R., ... François, O. (2012). Forecasting changes in population genetic structure of alpine plants in response to global warming. Molecular Ecology, 21, 2354-2368.

Koren, Y., Bell, R., \& Volinsky, C. (2009). Matrix factorization techniques for recommender systems. Computer, 42, 30-37.

Lee, D. D., \& Seung, H. S. (1999). Learning the parts of objects by non-negative matrix factorization. Nature, 401, 788-791.

Lee, C. R., Svardal, H., Farlow, A., Exposito-Alonso, M., Ding, W., Novikova, P., ... Nordborg, M. (2017). On the post-glacial spread of human commensal Arabidopsis thaliana. Nature Communications, 8, 14458.

Lipton, Z. C. (2018). The mythos of model interpretability. Queue, 16, 31-57.

Lotterhos, K. E., \& Whitlock, M. C. (2015). The relative power of genome scans to detect local adaptation depends on sampling design and statistical method. Molecular Ecology, 24, 1031-1046.

Lundemo, S., Falahati-Anbaran, M., \& Stenøien, H. K. (2009). Seed banks cause elevated generation times and effective population sizes of Arabidopsis thaliana in northern Europe. Molecular Ecology, 18, 2798-2811.

Manel, S., \& Holderegger, R. (2013). Ten years of landscape genetics. Trends in Ecology and Evolution, 28, 614-621.

Mardia, K.V., Kent, J.T., \& Bibby, J.M. (1979). Multivariate Analysis. Academic Press. 
Marchini J., \& Howie B. (2010). Genotype imputation for genome-wide association studies. Nature Reviews Genetics, 11, 499-511

Martins, H., Caye, K., Luu, K., Blum, M. G., \& François, O. (2016). Identifying outlier loci in admixed and in continuous populations using ancestral population differentiation statistics. Molecular Ecology, 25, 5029-5042.

McVean, G. (2009). A genealogical interpretation of principal components analysis. PLoS Genetics, 5, e1000686.

Murphy, K. P. (2012). Machine Learning: a Probabilistic Perspective. MIT press, Boston, USA.

Nychka, D., Furrer, R., Paige, J., \& Sain, S. (2017). fields: Tools for spatial data. University Corporation for Atmospheric Research, Boulder, CO, USA. doi: $10.5065 / \mathrm{D} 6 \mathrm{~W} 957 \mathrm{CT}$

Owen, A. B., \& Perry, P. O. (2009). Bi-cross-validation of the SVD and the nonnegative matrix factorization. The Annals of Applied Statistics, 3, 564-594.

Paradis, E., Gosselin, T., Grünwald, N. J., Jombart, T., Manel, S., \& Lapp, H. (2017). Towards an integrated ecosystem of $\mathrm{R}$ packages for the analysis of population genetic data. Molecular Ecology Resources, 17, 1-4

Patterson, N., Price, A. L., \& Reich, D. (2006). Population structure and eigenanalysis. PLoS Genetics, 2, e190.

Pauls, S. U., Nowak, C., Bálint, M., \& Pfenninger, M. (2013). The impact of global 
climate change on genetic diversity within populations and species. Molecular Ecology, 22, 925-946.

Pritchard, J. K., Stephens, M., \& Donnelly, P. (2000). Inference of population structure using multilocus genotype data. Genetics, 155, 945-959.

Razgour, O., Forester, B., Taggart, J. B., Bekaert, M., Juste, J., Ibáñez, C., ... Manel, S. (2019). Considering adaptive genetic variation in climate change vulnerability assessment reduces species range loss projections. Proceedings of the National Academy of Sciences, 116, 10418-10423.

Rellstab, C., Gugerli, F., Eckert, A. J., Hancock, A. M., \& Holderegger, R. (2015). A practical guide to environmental association analysis in landscape genomics. Molecular Ecology, 24, 4348-4370.

Rellstab, C., Zoller, S., Walthert, L., Lesur, I., Pluess, A. R., Graf, R., ... Gugerli, F. (2016). Signatures of local adaptation in candidate genes of oaks (Quercus spp.) with respect to present and future climatic conditions. Molecular Ecology, 25, 59075924.

Savolainen, O., Lascoux, M., \& Merilä, J. (2013). Ecological genomics of local adaptation. Nature Reviews Genetics, 14, 807-820.

Scheet, P., \& Stephens, M. (2006). A fast and flexible statistical model for largescale population genotype data: applications to inferring missing genotypes and haplotypic phase. The American Journal of Human Genetics, 78, 629-644.

Schoville, S. D., Bonin, A., François, O., Lobreaux, S., Melodelima, C., \& Manel, S. 
(2012). Adaptive genetic variation on the landscape: methods and cases. Annual Review of Ecology, Evolution and Systematics, 43, 23-43.

Skoglund, P., Sjödin, P., Skoglund, T., Lascoux, M., \& Jakobsson, M. (2014). Investigating population history using temporal genetic differentiation. Molecular Biology and Evolution, 31, 2516-2527.

Slatkin, M. (1991). Inbreeding coefficients and coalescence times. Genetics Research, $58,167-175$.

Sork, V. L., Davis, F. W., Westfall, R., Flint, A., Ikegami, M., Wang, H., \& Grivet, D. (2010). Gene movement and genetic association with regional climate gradients in California valley oak (Quercus lobata Née) in the face of climate change. Molecular Ecology, 19, 3806-3823.

Sork, V. L., Aitken, S. N., Dyer, R. J., Eckert, A. J., Legendre, P., \& Neale, D. B. (2013). Putting the landscape into the genomics of trees: approaches for understanding local adaptation and population responses to changing climate. Tree Genetics $\&$ Genomes, 9, 901-911.

Stekhoven, D. J., \& Bühlmann, P. (2012). MissForest: non-parametric missing value imputation for mixed-type data. Bioinformatics, 28, 112-118.

Storey, J., \& Tibshirani, R. (2003). Statistical significance for genomewide studies. Proceedings of the National Academy of Sciences USA, 100, 9440-9445.

Van Buuren, S. (2018). Flexible Imputation of Missing Data. CRC Press. 
Vatsiou, A. I., Bazin, E., \& Gaggiotti, O. E. (2016). Detection of selective sweeps in structured populations: a comparison of recent methods. Molecular Ecology, 25, 89-103.

Waldvogel, A. M., Feldmeyer, B., Rolshausen, G., Exposito-Alonso, M., Rellstab, C., Kofler, R., ... Savolainen, O. (2020). Evolutionary genomics can improve prediction of species' responses to climate change. Evolution Letters, 4, 4-18.

Data availability. The R package LEA 3.0 and higher versions are available from Bioconductor: www.bioconductor.org/packages/release/bioc/html/LEA.html. Development versions are available from www.bioconductor.org/packages/devel/ bioc/html/LEA.html, and github.com/bcm-uga/LEA. The computer scripts and the data used in the examples or in the simulation studies of this manuscript are available from a GitHub repository github.com/bcm-uga/LEA3_simulation_script under GNU General Public License v3.0.

Author contributions. OF designed research, CG and OF performed research, analyzed data and wrote the paper. 